\title{
The Existence of Positive Nonconstant Steady States in a Reaction: Diffusion Epidemic Model
}

\author{
Yuan Yuan, ${ }^{1}$ Hailing Wang, ${ }^{2}$ and Weiming Wang ${ }^{1}$ \\ ${ }^{1}$ College of Mathematics and Information Science, Wenzhou University, Wenzhou 325035, China \\ ${ }^{2}$ Department of Mathematics, Hubei Minzu University, Enshi, Hubei 445000, China \\ Correspondence should be addressed to Weiming Wang; weimingwang2003@163.com
}

Received 19 November 2013; Accepted 1 December 2013

Academic Editor: Kaifa Wang

Copyright (c) 2013 Yuan Yuan et al. This is an open access article distributed under the Creative Commons Attribution License, which permits unrestricted use, distribution, and reproduction in any medium, provided the original work is properly cited.

\begin{abstract}
We investigate the disease's dynamics of a reaction-diffusion epidemic model. We first give a priori estimates of upper and lower bounds for positive solutions to model and then give the conditions of the existence and nonexistence of the positive nonconstant steady states, which guarantees the existence of the stationary patterns.
\end{abstract}

\section{Introduction}

Infectious diseases are the second leading cause of death worldwide, after heart disease, and are responsible for more deaths annually than cancer [1]. Since the pioneer work of Kermark and McKendrick [2], mathematical models have been contributing to improve our understanding of infectious disease dynamics and help us develop preventive measures to control infection spread qualitatively and quantitatively.

Many studies indicate that spatial epidemiology with selfdiffusion has become a principal scientific discipline aiming at understanding the causes and consequences of spatial heterogeneity in disease transmission [3]. In these studies, reaction-diffusion equations have been intensively used to describe spatiotemporal dynamics. In particular, the spatial spread of infections has been studied by analyzing traveling wave solutions and calculating spread rates [4-10].

Besides, there has been some research on pattern formation in the spatial epidemic model, starting with Turing's seminal paper [11]. Turing's revolutionary idea was that the passive diffusion could interact with chemical reaction in such a way that even if the reaction by itself has no symmetrybreaking capabilities, diffusion can destabilize the symmetric solutions with the result that the system with diffusion has them [12]. In these studies [3,13-20], via standard linear analysis, the authors obtained the conditions of Turing instability, and, via numerical simulation, they showed the pattern formation induced by self-diffusion or cross-diffusion and found that model dynamics exhibits a diffusion controlled formation growth to stripes, spots, and coexistence or chaos pattern replication.

Recently, the researchers are interested in research on the stationary patterns due to the existence and nonexistence nonconstant solutions of the reaction-diffusion model [2129]. But the research on the existence and nonexistence nonconstant solutions of reaction-diffusion epidemic model, seems rare [3].

In this paper, we will focus on the disease's dynamics through studying the existence of the constant and nonconstant steady states of a simple reaction-diffusion epidemic model.

The rest of this paper is organized as follows. In Section 2, we derive a reaction-diffusion epidemic model. In Section 3, we give a priori estimates of upper and lower bounds for positive solutions to model. In Section 4, we give the main results on the existence and nonexistence of positive nonconstant steady states of the model. The paper ends with a brief discussion in Section 5.

\section{Basic Model}

In [30], Berezovsky and coworkers introduced a simple epidemic model through the incorporation of variable population, disease induced mortality, and emigration into the 
classic model of Kermark and McKendrick [2]. The total population $(N)$ is divided into two groups susceptible $(S)$ and infectious ( $I$ ); that is, $N=S+I$. The model describing the relations between the state variables is

$$
\begin{gathered}
\frac{d S}{d t}=r N\left(1-\frac{N}{K}\right)-\beta \frac{S I}{N}-(\mu+\theta) S, \\
\frac{d I}{d t}=\beta \frac{S I}{N}-(\mu+d) I,
\end{gathered}
$$

where the birth process incorporates density dependent effects via a logistic equation with the intrinsic growth rate $r$ and the carrying capacity $K ; S(t), I(t)$ represent population densities of susceptible and infected population, respectively; $\beta$ denotes the transmission rate (the infection rate constant); $\mu$ is the natural mortality; $d$ denotes the disease-induced mortality; $\theta$ is the per-capita emigration rate of noninfective.

For model (1), the epidemic threshold of basic reproduction number $R_{0}$ is then computed as

$$
R_{0}=\frac{\beta}{\mu+d} .
$$
by

The basic demographic reproductive number $R_{d}$ is given

$$
R_{d}=\frac{r}{\mu+\theta} .
$$

For simplicity, rescalling the model (1) by letting $S \rightarrow$ $S / K, I \rightarrow I / K$, and $t \rightarrow t /(\mu+d)$ leads to the following model:

$$
\begin{gathered}
\frac{d S}{d t}=\nu R_{d}(S+I)(1-(S+I))-R_{0} \frac{S I}{S+I}-\nu S, \\
\frac{d I}{d t}=R_{0} \frac{S I}{S+I}-I,
\end{gathered}
$$

where $v=(\mu+\theta) /(\mu+d)$ defined by the ratio of the average life-span of susceptibles to that of infections and $S+I \leq 1$.

See [30] for more details.

Assume that the habitat $\Omega \subset \mathbb{R}^{m}(m \geq 1)$ is a bounded domain with smooth boundary $\partial \Omega$ (when $m>1$ ), and $\mathbf{n}$ is the outward unit normal vector on $\partial \Omega$. We consider the following reaction-diffusion $S I$ epidemic model:

$$
\begin{aligned}
& \frac{\partial S}{\partial t}-d_{S} \Delta S=v R_{d}(S+I)(1-(S+I)) \\
& -R_{0} \frac{S I}{S+I}-v S, \quad x \in \Omega, t>0, \\
& \frac{\partial I}{\partial t}-d_{I} \Delta I=R_{0} \frac{S I}{S+I}-I, \quad x \in \Omega, t>0, \\
& S(x, 0)=S_{0}(x)>0, \quad I(x, 0)=I_{0}(x) \geq 0, x \in \Omega, \\
& \frac{\partial S}{\partial \mathbf{n}}=\frac{\partial I}{\partial \mathbf{n}}=0, \quad x \in \partial \Omega, t>0,
\end{aligned}
$$

where the nonnegative constants $d_{S}$ and $d_{I}$ are the diffusion coefficients of $S$ and $I$, respectively. The symbol $\Delta$ is the Laplacian operator. The homogeneous Neumann boundary condition implies that the above model is self-contained and there is no infection across the boundary.

The corresponding kinetic model (5) with $m=2$ has been investigated by Wang et al. [20].

In this paper, we concentrated on the steady states of model (5) which satisfy

$$
\begin{gathered}
-d_{S} \Delta S=v R_{d}(S+I)(1-(S+I))-R_{0} \frac{S I}{S+I}-v S, \quad x \in \Omega, \\
-d_{I} \Delta I=R_{0} \frac{S I}{S+I}-I, \quad x \in \Omega \\
\frac{\partial S}{\partial \mathbf{n}}=\frac{\partial I}{\partial \mathbf{n}}=0, \quad x \in \partial \Omega .
\end{gathered}
$$

Throughout this paper, the positive solution $(S, I)$ satisfying model (6) refers to a classical one with $S>0, I>0$ on $\bar{\Omega}$. Clearly, model (6) has a unique positive constant solution (endemic equilibrium) $E^{*}=\left(S^{*}, I^{*}\right)$ if $R_{d}>\left(\nu+R_{0}-1\right) / R_{0} \nu$ and $R_{0}>1$, where

$$
S^{*}=\frac{\nu R_{0} R_{d}-R_{0}+1-\nu}{\nu R_{0}^{2} R_{d}}, \quad I^{*}=\left(R_{0}-1\right) S^{*} .
$$

\section{A Priori Estimates for Positive Solutions to Model (6)}

The main purpose of this section is to give a priori upper and lower positive bounds for positive solution of model (6). To this aim, we first cite two known results. The first is due to Lin et al. [31] and the second to Lou and $\mathrm{Ni}$ [32]. In the following, let us denote the constants $\nu, R_{d}$, and $R_{0}$ collectively by $\Lambda$. The positive constants $C, \underline{C}, \bar{C}, C^{*}$, and so forth will depend only on the domains $\Omega$ and $\Lambda$.

Lemma 1 (Harnack inequality [31]). Let $w \in C^{2}(\Omega) \cap C^{1}(\bar{\Omega})$ be a positive solution to $\Delta w(x)+c(x) w(x)=0$, where $\in C(\bar{\Omega})$, satisfying the homogeneous Neumann boundary conditions. Then there exists a positive constant $C^{*}=C^{*}\left(\|c\|_{\infty}, \Omega\right)$, such that

$$
\max _{\bar{\Omega}} w \leq C^{*} \min _{\bar{\Omega}} w
$$

Lemma 2 (maximum principle [32]). Let $\Omega$ be a bounded Lipschitz domain in $\mathbb{R}^{m}$ and $g \in C(\bar{\Omega} \times \mathbb{R})$.

(a) Assume that $w \in C^{2}(\Omega) \cap C^{1}(\bar{\Omega})$ and satisfies

$$
\begin{gathered}
\Delta w(x)+g(x, w(x)) \geq 0, \quad x \in \Omega, \\
\frac{\partial w}{\partial \mathbf{n}} \leq 0, \quad x \in \partial \Omega .
\end{gathered}
$$

$$
\text { If } w\left(x_{0}\right)=\max _{\bar{\Omega}} w(x) \text {, then } g\left(x_{0}, w\left(x_{0}\right)\right) \geq 0 \text {. }
$$


(b) Assume that $w \in C^{2}(\Omega) \cap C^{1}(\bar{\Omega})$ and satisfies

$$
\begin{gathered}
\Delta w(x)+g(x, w(x)) \leq 0, \quad x \in \Omega, \\
\frac{\partial w}{\partial \mathbf{n}} \geq 0, \quad x \in \partial \Omega .
\end{gathered}
$$

$$
\text { If } w\left(x_{1}\right)=\min { }_{\Omega} w(x) \text {, then } g\left(x_{1}, w\left(x_{1}\right)\right) \leq 0 \text {. }
$$

Theorem 3. If $R_{0}>1$, then the positive solution $(S(x), I(x))$ of model (6) satisfies

$$
\max _{\bar{\Omega}} S(x)<\frac{1}{4} R_{d}, \quad \max _{\bar{\Omega}} I(x)<\frac{1}{4} R_{d}\left(R_{0}-1\right) .
$$

Proof. Assume that $(S(x), I(x))$ is a positive solution of model (6). We set

$$
S\left(x_{1}\right)=\max _{\bar{\Omega}} S(x), \quad I\left(x_{2}\right)=\max _{\bar{\Omega}} I(x) .
$$

By applying Lemma 2, we have

$$
\begin{aligned}
\frac{1}{4} R_{d} & -\nu S\left(x_{1}\right) \\
& \geq \nu R_{d}\left(S\left(x_{1}\right)+I\left(x_{1}\right)\right)\left(1-\left(S\left(x_{1}\right)+I\left(x_{1}\right)\right)\right)-\nu S\left(x_{1}\right) \\
& \geq \frac{R_{0} S\left(x_{1}\right) I\left(x_{1}\right)}{S\left(x_{1}\right)+I\left(x_{1}\right)}>0,
\end{aligned}
$$

and $R_{0} S\left(x_{2}\right) I\left(x_{2}\right) /\left(S\left(x_{2}\right)+I\left(x_{2}\right)\right) \geq I\left(x_{2}\right)$. This clearly gives

$$
S\left(x_{1}\right)<\frac{1}{4} R_{d}, \quad I\left(x_{2}\right) \leq\left(R_{0}-1\right) S\left(x_{2}\right)<\frac{1}{4} R_{d}\left(R_{0}-1\right) .
$$

Theorem 4. Assume that $R_{d}>1$ and $R_{0}>1$. Let $d$ and $D$ be fixed positive constants. Then there exists a positive constant $\underline{C}=\underline{C}(\Lambda, d)$ such that, if $d_{S}, d_{I}>d$, every positive solution $\overline{(S}(x), \bar{I}(x))$ of model (6) satisfies

$$
\min _{\bar{\Omega}} S(x)>\underline{C}, \quad \min _{\bar{\Omega}} I(x)>\underline{C} .
$$

Proof. Let

$$
\begin{gathered}
c_{1}(x)=\frac{1}{d_{S}}\left(\nu R_{d}\left(1+\frac{I}{S}\right)(1-(S+I))-\frac{R_{0} I}{S+I}-\nu\right), \\
c_{2}(x)=\frac{1}{d_{I}}\left(\frac{R_{0} S}{S+I}-1\right) .
\end{gathered}
$$

In view of Theorem 3 , there exists a positive constant $C=$ $C(\Lambda)$ such that $\left\|c_{1}(x)\right\|_{\infty} \leq C,\left\|c_{2}(x)\right\|_{\infty} \leq C$ provided that $d_{S}, d_{I}>d$. As $S$ and $I$ satisfy

$$
\begin{aligned}
& \Delta S(x)+c_{1}(x) S=0, \quad x \in \Omega, \\
& \Delta I(x)+c_{2}(x) I=0, \quad x \in \Omega, \\
& \frac{\partial S}{\partial \mathbf{n}}=\frac{\partial I}{\partial \mathbf{n}}=0, \quad x \in \partial \Omega .
\end{aligned}
$$

It follows from Lemma 1 that there exists a positive constant $C^{*}=C^{*}(\Lambda, d)$ such that

$$
\max _{\bar{\Omega}} S \leq C^{*} \min _{\bar{\Omega}} S, \quad \max _{\bar{\Omega}} I \leq C^{*} \min _{\bar{\Omega}} I
$$

for $d_{S}, d_{I} \geq d$.

Now, on the contrary, suppose that (15) is not true, then there exist sequences $\left\{d_{S, i}\right\}_{i=1}^{\infty},\left\{d_{I, i}\right\}_{i=1}^{\infty}$ with $\left(d_{S, i}, d_{I, i}\right) \in$ $[d, \infty) \times[d, \infty)$ and the positive solution $\left(S_{i}, I_{i}\right)$ of model $(6)$ corresponding to $\left(d_{S}, d_{I}\right)=\left(d_{S, i}, d_{I, i}\right)$, such that

$$
\min _{\bar{\Omega}} S_{i}(x) \longrightarrow 0 \quad \text { or } \min _{\bar{\Omega}} I_{i}(x) \longrightarrow 0 \quad \text { as } i \longrightarrow \infty \text {. }
$$

It follows from Lemma 1 that

$$
\begin{aligned}
& S_{i}(x) \longrightarrow 0 \text { or } I_{i}(x) \longrightarrow 0 \\
& \text { uniformly on } \bar{\Omega} \text { as } i \longrightarrow \infty \text {. }
\end{aligned}
$$

$\left(S_{i}, I_{i}\right)$ satisfies

$$
\begin{aligned}
-d_{S, i} \Delta S_{i}= & \nu R_{d}\left(S_{i}+I_{i}\right)\left(1-\left(S_{i}+I_{i}\right)\right) \\
& -\frac{R_{0} S_{i} I_{i}}{S_{i}+I_{i}}-\nu S_{i}, \quad x \in \Omega, \\
-d_{I, i} \Delta I_{i}= & \frac{R_{0} S_{i} I_{i}}{S_{i}+I_{i}}-I_{i}, \quad x \in \Omega, \\
\frac{\partial S_{i}}{\partial \mathbf{n}}=\frac{\partial I_{i}}{\partial \mathbf{n}}= & 0, \quad x \in \partial \Omega .
\end{aligned}
$$

Integrating by parts, we obtain that, for $i=1,2, \ldots$,

$$
\begin{gathered}
\int_{\Omega}\left(\nu R_{d}\left(S_{i}+I_{i}\right)\left(1-\left(S_{i}+I_{i}\right)\right)-\frac{R_{0} S_{i} I_{i}}{S_{i}+I_{i}}-\nu S_{i}\right) d x=0, \\
\int_{\Omega} I_{i}\left(\frac{R_{0} S_{i}}{S_{i}+I_{i}}-1\right) d x=0 .
\end{gathered}
$$

By the regularity theory for elliptic equations [33], we see that there exist a subsequence of $\left\{\left(S_{i}, I_{i}\right)\right\}_{i}^{\infty}$, which we will still denote by $\left\{\left(S_{i}, I_{i}\right)\right\}_{i}^{\infty}$, and two nonnegative functions $\widetilde{S}, \widetilde{I} \in$ $C^{2}(\Omega)$, such that $\left(S_{i}, I_{i}\right) \rightarrow(\widetilde{S}, \widetilde{I})$ in $\left[C^{2}(\Omega)\right]^{2}$ as $i \rightarrow \infty$. By (20), we have that $\widetilde{S} \equiv 0$ or $\widetilde{I} \equiv 0$.

Letting $i \rightarrow \infty$ in (22) we obtain that

$$
\begin{gathered}
\int_{\Omega}\left(\nu R_{d}(\widetilde{S}+\widetilde{I})(1-(\widetilde{S}+\widetilde{I}))-\frac{R_{0} \widetilde{S} \widetilde{I}}{\widetilde{S}+\widetilde{I}}-v \widetilde{S}\right) d x=0, \\
\int_{\Omega} \widetilde{I}\left(\frac{R_{0} \widetilde{S}}{\widetilde{S}+\widetilde{I}}-1\right) d x=0 .
\end{gathered}
$$

Case $1(\widetilde{S} \equiv 0, \widetilde{I} \not \equiv 0$ or $\widetilde{S} \equiv 0, \widetilde{I} \equiv 0)$. Since $I_{i}$ satisfies the second inequality of (18), $I_{i}>0$ on $\bar{\Omega}$. Therefore, $R_{0} S_{i} /\left(S_{i}+\right.$ $\left.I_{i}\right)-1 \rightarrow-1<0$ on $\bar{\Omega}$ as $i \rightarrow \infty$. Hence, $\int_{\Omega} I_{i}\left(R_{0} S_{i} /\left(S_{i}+\right.\right.$ $\left.\left.I_{i}\right)-1\right) d x<0$ for sufficiently large $i$ which contradicts the second integral identity of (22). 
Case $2(\widetilde{I} \equiv 0, \widetilde{S} \neq 0)$. As above, $\widetilde{S}>0$ on $\bar{\Omega}$. It follows from the first integral identity of (23) that

$$
\int_{\Omega} \widetilde{S}\left(\nu R_{d}(1-\widetilde{S})-v\right) d x=0 .
$$

This fact combines with $0<\widetilde{S} \leq(1 / 4) R_{d}$ yielding to $\widetilde{S}=$ $1-1 / R_{d}$, which implies that $R_{0} S_{i} /\left(S_{i}+I_{i}\right) \rightarrow R_{0}$ uniformly on $\bar{\Omega}$ as $i \rightarrow \infty$, since $I_{i} \rightarrow 0$ uniformly on $\bar{\Omega}$. As $R_{0}>1$, this contradicts the second integral identity of (23) and the fact that $I_{i}>0$. This completes the proof.

\section{Existence and Nonexistence of Positive Nonconstant Steady States}

In this section, we provide some sufficient conditions for the existence and nonexistence of nonconstant positive solution of model (6) by using the Leray-Schauder degree theory [34]. From now on, we denote by

$$
0=\mu_{0}<\mu_{1}<\mu_{2}<\mu_{3}<\cdots
$$

the eigenvalues of the operator $-\Delta$ on $\Omega$ with the zero-flux boundary conditions.

4.1. Nonexistence for Positive Nonconstant Steady States to Model (6). This section is devoted to the consideration of the nonexistence for the nonconstant positive solutions of model (6), and, in the following results, the diffusion coefficients do play a significant role.

Theorem 5. Assume that $R_{0}>1$. Let $D_{2}$ be a fixed positive constant with $D_{2}>\left(R_{0}-1\right) / \mu_{1}$. Then there exists a positive constant $D_{1}\left(\Lambda, D_{2}\right)$ such that model (6) has no positive nonconstant solution provided that $d_{S} \geq D_{1}$ and $d_{I} \geq D_{2}$.

Proof. Let $(S(x), I(x))$ be any positive solution of model (6) and denote $\bar{g}=|\Omega|^{-1} \int_{\Omega} g d x$. Then, multiplying the first equation of model (6) by $(S-\bar{S})$, integrating over $\Omega$, by virtue of Theorem 3, we have that

$$
\begin{aligned}
d_{S} \int_{\Omega}|\nabla S|^{2} d x \\
=\int_{\Omega}(S-\bar{S}) \\
\quad \times\left(\nu R_{d}(S+I)(1-(S+I))-\frac{R_{0} S I}{S+I}-\nu S\right) d x \\
=\int_{\Omega}(S-\bar{S})^{2} \\
\quad \times\left(\nu\left(R_{d}-1\right)-v R_{d}(S+\bar{S})+2 v R_{d} I-\frac{R_{0} I \bar{I}}{(\bar{S}+\bar{I})(S+I)}\right) d x
\end{aligned}
$$

$$
\begin{aligned}
&+ \int_{\Omega}\left(\nu R_{d}(1-(I+\bar{I})+2 \bar{S})-\frac{R_{0} S \bar{S}}{(\bar{S}+\bar{I})(S+I)}\right) \\
& \times(S-\bar{S})(I-\bar{I}) d x, \\
& \leq C_{1} \int_{\Omega}(S-\bar{S})^{2}+C_{2} \int_{\Omega}|S-\bar{S}||I-\bar{I}| d x,
\end{aligned}
$$

where $C_{1}, C_{2}$ depend only on $\Lambda$. In a similar manner, we multiply the second equation in model (6) by $(I-\bar{I})$ to have

$$
\begin{aligned}
& d_{I} \int_{\Omega}|\nabla I|^{2} d x \\
& \quad=\int_{\Omega}(I-\bar{I})\left(\frac{R_{0} S I}{S+I}-I\right) d x \\
& \quad=\int_{\Omega}(I-\bar{I})^{2}\left(-1+\frac{R_{0} \bar{S} S}{(\bar{S}+\bar{I})(S+I)}\right) d x \\
& \quad+\int_{\Omega} \frac{R_{0} \bar{I} I}{(\bar{S}+\bar{I})(S+I)}(S-\bar{S})(I-\bar{I}) d x \\
& \quad\left(R_{0}-1\right) \int_{\Omega}(I-\bar{I})^{2} d x \\
& \quad+R_{0} \int_{\Omega}|S-\bar{S}||I-\bar{I}| d x .
\end{aligned}
$$

It follows from (26), (27) and the $\varepsilon$-Young inequality that

$$
\begin{aligned}
& \int_{\Omega}\left(d_{S}|\nabla S|^{2}+d_{I}|\nabla I|^{2}\right) d x \\
& \leq \int_{\Omega}\left(\left(C_{1}+\frac{C}{2 \varepsilon}\right)(S-\bar{S})^{2}+\left(R_{0}-1+\frac{\varepsilon C}{2}\right)(I-\bar{I})^{2}\right) d x,
\end{aligned}
$$

where $C=C_{2}+R_{0}$. It follows from the well-known Poincaré inequality that

$$
\begin{aligned}
\int_{\Omega} & \left(d_{S}|\nabla S|^{2}+d_{I}|\nabla I|^{2}\right) d x \\
\leq & \frac{1}{\mu_{1}}\left(C_{1}+\frac{C}{2 \varepsilon}\right) \int_{\Omega}|\nabla S|^{2} d x \\
& +\frac{1}{\mu_{1}}\left(R_{0}-1+\frac{\varepsilon C}{2}\right) \int_{\Omega}|\nabla I|^{2} d x .
\end{aligned}
$$

Since $d_{I} \mu_{1}>R_{0}-1$ from the assumption, we can find a sufficiently small $\varepsilon_{0}$ such that $d_{I} \mu_{1} \geq R_{0}-1+\varepsilon C / 2$. Finally, by taking $D_{1}:=\left(1 / \mu_{1}\right)\left(C_{1}+C / 2 \varepsilon_{0}\right)$ one can conclude that $S=\bar{S}$ and $I=\bar{I}$, which asserts our results.

4.2. Existence for Positive Nonconstant Steady States to Model (6). In this section, we discuss the global existence of nonconstant positive classical solutions to model (6), which guarantees the existence of the stationary patterns $[21,24,26$, 27]. 
Unless otherwise specified, in this section, we always require that $R_{d}>\left(\nu+R_{0}-1\right) / R_{0} v$ and $R_{0}>1$, which guarantees that model (6) has one positive constant steady state $E^{*}$. From now on, let us denote

$$
\begin{gathered}
\mathbf{u}=(S, I), \\
\mathbf{u}^{*}=\left(S^{*}, I^{*}\right)=\left(\frac{\nu R_{0} R_{d}-R_{0}+1-\nu}{\nu R_{0}^{2} R_{d}},\left(R_{0}-1\right) S^{*}\right) .
\end{gathered}
$$

Let $\mathbf{X}=\left\{\mathbf{u} \in\left[C^{2}(\Omega)\right]^{2} \mid \partial \mathbf{u} / \partial \mathbf{n}=0, x \in \partial \Omega\right\}$ and $\mathbf{X}^{+}=$ $\{\mathbf{u} \in \mathbf{X} \mid S, I>0, x \in \bar{\Omega}\}$. Then we write model (6) in the form

$$
\begin{aligned}
-\Delta \mathbf{u} & =\mathbf{G}(\mathbf{u}), \quad x \in \Omega, \\
\frac{\partial \mathbf{u}}{\partial \mathbf{n}} & =0, \quad x \in \partial \Omega,
\end{aligned}
$$

where

$$
\mathbf{G}(\mathbf{u})=\left(\begin{array}{c}
\frac{1}{d_{S}}\left(\nu R_{d}(S+I)(1-(S+I))-\frac{R_{0} S I}{S+I}-\nu S\right) \\
\frac{1}{d_{I}}\left(\frac{R_{0} S I}{S+I}-I\right)
\end{array}\right) .
$$

Define a compact operator $\mathscr{F}: \mathbf{X}^{+} \rightarrow \mathbf{X}^{+}$by

$$
\mathscr{F}(\mathbf{u}):=(\mathbf{I}-\Delta)^{-1}\{\mathbf{G}(\mathbf{u})+\mathbf{u}\},
$$

where $(\mathbf{I}-\Delta)^{-1}$ is the inverse operator of $\mathbf{I}-\Delta$ subject to the zero-flux boundary condition. Then $\mathbf{u}$ is a positive solution of model (31) if and only if $\mathbf{u}$ satisfies

$$
(\mathbf{I}-\mathscr{F}) \mathbf{u}=0, \quad x \in \Omega .
$$

To apply the index theory, we investigate the eigenvalue of the problem

$$
-\left(\mathbf{I}-\mathscr{F}_{\mathbf{u}}\left(\mathbf{u}^{*}\right)\right) \Psi=\lambda \Psi, \quad \Psi \neq \mathbf{0},
$$

where $\Psi=\left(\Psi_{1}, \Psi_{2}\right)^{T}$ and $\mathscr{F}_{\mathbf{u}}\left(\mathbf{u}^{*}\right)=(\mathbf{I}-\Delta)^{-1}(\mathbf{I}+\mathbf{A})$ with

$$
\begin{aligned}
\mathbf{A} & =\left(\begin{array}{cc}
\frac{4 R_{0}+2 \nu-R_{0}^{2}-\nu R_{0} R_{d}-\nu R_{0}-3}{d_{S} R_{0}} & -\frac{v R_{0} R_{d}+3-2 R_{0}-2 \nu}{d_{S} R_{0}} \\
\frac{\left(R_{0}-1\right)^{2}}{d_{I} R_{0}} & -\frac{R_{0}-1}{d_{I} R_{0}}
\end{array}\right) \\
& :=\left(\begin{array}{cc}
d_{S}^{-1} a_{1} & -d_{S}^{-1} a_{2} \\
d_{I}^{-1} a_{3} & -d_{I}^{-1} a_{4}
\end{array}\right) .
\end{aligned}
$$

$\lambda$ is an eigenvalue of (35) if and only if $\lambda$ is an eigenvalue of the matrix $\left(\mu_{i}+1\right)^{-1}\left(\mu_{i} \mathbf{I}-\mathbf{A}\right)$ for any $i \geq 0$. Therefore, $\mathbf{I}-\mathscr{F}_{\mathbf{u}}\left(\mathbf{u}^{*}\right)$ is invertible if and only if, for any $i \geq 0$, the matrix

$$
M_{i}:=\mu_{i} \mathbf{I}-\mathbf{A}=\left(\begin{array}{cc}
\mu_{i}-d_{S}^{-1} a_{1} & d_{S}^{-1} a_{2} \\
-d_{I}^{-1} a_{3} & \mu_{i}+d_{I}^{-1} a_{4}
\end{array}\right)
$$

is invertible. A straightforward computation yields

$$
\begin{aligned}
\operatorname{det}\left(M_{i}\right)= & d_{S}^{-1} d_{I}^{-1} \\
& \times\left(d_{S} d_{I} \mu_{i}^{2}+\left(d_{S} a_{4}-d_{I} a_{1}\right) \mu_{i}+\rho\right),
\end{aligned}
$$

where $\rho=\left(1 / R_{0}\right)\left(\nu R_{0} R_{d}-R_{0}+1-\nu\right)\left(R_{0}-1\right)>0$. For the sake of convenience, we denote

$$
H\left(d_{S}, d_{I}, \mu\right)=d_{S} d_{2} \mu_{i}^{2}+\left(d_{S} a_{4}-d_{I} a_{1}\right) \mu_{i}+\rho
$$

Then $H\left(d_{S}, d_{I}, \mu\right)=d_{S} d_{2} \operatorname{det}\left(M_{i}\right)$.
If $\left(d_{S} a_{4}-d_{I} a_{1}\right)^{2}>4 d_{S} d_{2} \rho$, then $H\left(d_{S}, d_{I}, \mu\right)=0$ has two real roots $\mu^{ \pm}$given by

$$
\begin{aligned}
& \mu^{+}\left(d_{S}, d_{I}\right) \\
& \quad=\frac{1}{2 d_{S} d_{2}}\left(d_{I} a_{1}-d_{S} a_{4}+\sqrt{\left(d_{S} a_{4}-d_{I} a_{1}\right)^{2}-4 d_{S} d_{2} \rho}\right), \\
& \mu^{-}\left(d_{S}, d_{I}\right) \\
& \quad=\frac{1}{2 d_{S} d_{2}}\left(d_{I} a_{1}-d_{S} a_{4}-\sqrt{\left(d_{S} a_{4}-d_{I} a_{1}\right)^{2}-4 d_{S} d_{2} \rho}\right) .
\end{aligned}
$$

Set $B:=B\left(d_{S}, d_{I}\right)=\left\{\mu: \mu \geq 0, \mu^{-}\left(d_{S}, d_{I}\right)<\mu<\right.$ $\left.\mu^{+}\left(d_{S}, d_{I}\right)\right\}, S_{p}=\left\{\mu_{0}, \mu_{1}, \mu_{2}, \ldots\right\}$, and $m\left(\mu_{i}\right)$ the multiplicity of $\mu_{i}$.

To compute index $\left(\mathbf{I}-\mathscr{F}, \mathbf{u}^{*}\right)$, we can assert the following conclusion by Pang and Wang [22].

Lemma 6 (see [22]). Suppose $H\left(d_{S}, d_{I}, \mu_{i}\right) \neq 0$ for all $\mu_{i} \in S_{p}$. Then

$$
\text { index }\left(\mathbf{I}-\mathscr{F}, \mathbf{u}^{*}\right)=(-1)^{\sigma} \text {, }
$$


where

$$
\sigma= \begin{cases}\sum_{\mu \in B \cap S_{j}} m\left(u_{i}\right), & \text { if } B \cap S_{p} \neq \emptyset \\ 0, & \text { if } B \cap S_{p}=\emptyset .\end{cases}
$$

In particular, if $H\left(d_{S}, d_{I}, \mu\right)>0$ for all $\mu \geq 0$, then $\sigma=0$.

From Lemma 6, we see that to calculate the index of index $\left(\mathbf{I}-\mathscr{F}, \mathbf{u}^{*}\right)$, the key step is to determine the range of $\mu$ for which $H\left(d_{S}, d_{I}, \mu\right)<0$.

Theorem 7. Assume that $R_{d}>\max \left\{1,\left(\nu+R_{0}-1\right) / R_{0} \nu\right\}$. If $4 R_{0}+2 v-R_{0}^{2}-v R_{0} R_{d}-v R_{0}-3>0,\left(4 R_{0}+2 v-R_{0}^{2}-v R_{0} R_{d}-\right.$ $\left.\nu R_{0}-3\right) / d_{S} R_{0} \in\left(\mu_{j}, \mu_{j+1}\right)$ for some $j \geq 1$, and $\sum_{i=1}^{j} m\left(\mu_{i}\right)$ is odd, then there exists a positive constant $d^{*}$ such that model (6) has at least one nonconstant solution if $d_{I}>d^{*}$.

Proof. Since $4 R_{0}+2 v-R_{0}^{2}-v R_{0} R_{d}-v R_{0}-3>0$, equivalently, $a_{1}>0$, it follows that if $d_{I}$ is large enough, then $\left(d_{S} a_{4}-d_{I} a_{1}\right)^{2}>4 d_{S} d_{I} \rho$ and $0<\mu^{-}\left(d_{S}, d_{I}\right)<\mu^{+}\left(d_{S}, d_{I}\right)$. Furthermore,

$$
\mu^{-}\left(d_{S}, d_{I}\right) \longrightarrow 0, \quad \mu^{+}\left(d_{S}, d_{I}\right) \longrightarrow \frac{a_{1}}{d_{S}}, \quad \text { as } d_{I} \longrightarrow \infty
$$

Since $a_{1} / d_{S} \in\left(\mu_{j}, \mu_{j+1}\right)$ for some $j \geq 1$, there exists $d_{0} \gg 1$ such that

$$
\begin{array}{r}
\mu^{+}\left(d_{S}, d_{I}\right) \in\left(\mu_{j}, \mu_{j+1}\right), \quad 0<\mu^{-}\left(d_{S}, d_{I}\right)<\mu_{1}, \\
\forall d_{I} \geq d_{0} .
\end{array}
$$

By Theorem 5, we know that there exists $d>d_{0}$ such that model (6) with diffusion coefficients $d_{S}=d$ and $d_{I} \geq d$ has no nonconstant solutions. Moreover, we can choose $d$ so large that $a_{1} / d<\mu_{1}$. It follows that there exists $d^{*}>d$ such that

$$
0<\mu^{-}\left(d_{S}, d_{I}\right)<\mu^{+}\left(d_{S}, d_{I}\right)<\mu_{1}, \quad \forall d_{I} \geq d^{*} .
$$

We shall prove that, for any $d_{I} \geq d^{*}$, model (6) has at least one nonconstant positive solution. On the contrary, suppose that this assertion is not true for some $d_{I}^{*}>d^{*}$. In the following, we will derive a contradiction by using a homotopy argument.

By virtue of Theorems 3 and 4, there exists a positive constant $C=C\left(\Lambda, d, d_{S}, d^{*}, d_{I}{ }^{*}\right)$ such that the positive solution $(S(x), I(x))$ of model (6) satisfies $C^{-1}<S, I<C$.

Set

$$
\mathscr{M}=\left\{(S, I) \in C(\bar{\Omega}) \times C(\bar{\Omega}): C^{-1}<S, I<C, x \in \bar{\Omega}\right\},
$$

and define

$$
\Phi: \mathscr{M} \times[0,1] \longrightarrow C(\bar{\Omega}) \times C(\bar{\Omega})
$$

by

$$
\Phi(\mathbf{u}, \theta)=(\mathbf{I}-\Delta)^{-1}\{\mathbf{G}(\mathbf{u}, \theta)+\mathbf{u}\}
$$

where

$$
\mathbf{G}(\mathbf{u}, \theta)
$$

$$
=\left(\begin{array}{c}
\left(\theta d_{S}+(1-\theta) d\right)^{-1}\left(\nu R_{d}(S+I)(1-(S+I))-\frac{R_{0} S I}{S+I}-\nu S\right) \\
\left(\theta d_{I}+(1-\theta) d^{*}\right)^{-1}\left(\frac{R_{0} S I}{S+I}-I\right)
\end{array}\right) .
$$

It is clear that finding the positive solution of model (31) is equivalent to finding the fixed point of $\Phi(\mathbf{u}, 1)$ in $\mathscr{M}$. Further, by virtue of the definition of $\mathscr{M}$, we have that $\Phi(\mathbf{u}, \theta)=0$ has no fixed point in $\partial \mathscr{M}$ for all $0 \leq \theta \leq 1$.

Since $\Phi(\mathbf{u}, t)$ is compact, the Leray-Schauder topological degree $\operatorname{deg}(\mathbf{I}-\Phi(\mathbf{u}, \theta), \mathscr{M}, 0)$ is well defined. From the invariance of Leray-Schauder degree at the homotopy, we deduce

$$
\operatorname{deg}(\mathbf{I}-\Phi(\mathbf{u}, 1), \mathscr{M}, 0)=\operatorname{deg}(\mathbf{I}-\Phi(\mathbf{u}, 0), \mathscr{M}, 0) .
$$

In view of $\mu^{-} \in\left(\mu_{i}, \mu_{i+1}\right)$ and $\mu^{+} \in\left(\mu_{j}, \mu_{j+1}\right)$, we have $B\left(d_{S}, d_{I}\right) \cap S_{j}=\left\{\mu_{i+1}, \mu_{i+2}, \ldots, \mu_{p}\right\}$. Clearly, $\mathbf{I}-\Phi(\mathbf{u}, 1)=\mathbf{I}-\mathscr{F}$. Thus, if model (6) has no other solutions except the constant one $\mathbf{u}^{*}$, then Lemma 6 shows that

$$
\begin{aligned}
& \operatorname{deg}(\mathbf{I}-\Phi(\mathbf{u}, 1), \mathscr{M}, 0) \\
& \quad=\operatorname{index}\left(\mathbf{I}-\mathscr{F}, \mathbf{u}^{*}\right)=(-1)^{\sum_{i=1}^{j} m\left(u_{i}\right)}=-1 .
\end{aligned}
$$

On the contrary, by the choice of $d$ and $d^{*}$, we have that $B\left(\bar{d}_{1}, \bar{d}_{2}\right) \cap S_{p}=\emptyset$ and $\mathbf{u}^{*}$ is the only fixed point of $\Phi(\mathbf{u}, 0)$. It therefore follows from Lemma 6 that

$$
\begin{aligned}
& \operatorname{deg}(\mathbf{I}-\Phi(\mathbf{u}, 0), \mathscr{M}, 0) \\
& \quad=\operatorname{index}\left(\mathbf{I}-\mathscr{F}, \mathbf{u}^{*}\right)=(-1)^{0}=1 .
\end{aligned}
$$

From (50)-(52), we get a contradiction. Therefore, there exists a nonconstant solution of model (6). The proof is completed.

\section{Discussion}

In this paper, we investigate the disease's dynamics through studying the existence and nonexistence positive constant steady states of a reaction-diffusion epidemic model. We give a priori estimates for positive solutions to model and show that the nonconstant positive steady states exist due to the emergence of diffusion, which demonstrates that stationary patterns can be found as a result of diffusion. The numerical results about the stationary patterns for model (5) can be found in [20].

On the other hand, there are plenty of papers which focus on the pattern formation of reaction-diffusion population models via standard linear analysis method and numerical simulations. But there is little literature analytically concerning the existence of a stationary patterns via theory and methods of partial differential equations infrequently. The methods and results in the present paper may enrich the research of pattern formation in the spatial epidemic model. 


\section{Conflict of Interests}

The authors declare that there is no conflict of interests regarding the publication of this paper.

\section{Acknowledgments}

The authors thank the anonymous referee for very helpful suggestions and comments which led to improvement of our original paper. This research was supported by the National Science Foundation of China (61373005) and Zhejiang Provincial Natural Science Foundation (LY12A01014).

\section{References}

[1] A. Lahrouz and A. Settati, "Asymptotic properties of switching diffusion epidemic model with varying population size," Applied Mathematics and Computation, vol. 219, no. 24, pp. 11134-11148, 2013.

[2] M. D. Kermark and A. G. Mckendrick, "Contributions to the mathematical theory of epidemics," Proceedings of the Royal Society of London A, vol. 115, pp. 700-721, 1927.

[3] Y. Cai, D. Chi, W. Liu, and W. Wang, "Stationary patterns of a cross-diffusion epidemic model," Abstract and Applied Analysis, vol. 2013, Article ID 852698, 10 pages, 2013.

[4] E. E. Holmes, M. A. Lewis, J. E. Banks, and R. R. Veit, "Partial differential equations in ecology: spatial interactions and population dynamics," Ecology, vol. 75, no. 1, pp. 17-29, 1994.

[5] Y. Hosono and B. Ilyas, "Traveling waves for a simple diffusive epidemic model," Mathematical Models and Methods in Applied Sciences, vol. 5, no. 7, pp. 935-966, 1995.

[6] B. T. Grenfell, O. N. Bjørnstad, and J. Kappey, "Travelling waves and spatial hierarchies in measles epidemics," Nature, vol. 414, no. 6865, pp. 716-723, 2001.

[7] A. L. Lloyd and V. A. A. Jansen, "Spatiotemporal dynamics of epidemics: synchrony in metapopulation models," Mathematical Biosciences, vol. 188, no. 1-2, pp. 1-16, 2004.

[8] K. Wang, W. Wang, and S. Song, "Dynamics of an HBV model with diffusion and delay," Journal of Theoretical Biology, vol. 253, no. 1, pp. 36-44, 2008.

[9] R. Xu and Z. Ma, "An HBV model with diffusion and time delay," Journal of Theoretical Biology, vol. 257, no. 3, pp. 499-509, 2009.

[10] S. Wang, W. Liu, Z. Guo, and W. Wang, "Traveling wave solutions in a reaction-diffusion epidemic model," Abstract and Applied Analysis, vol. 2013, Article ID 216913, 13 pages, 2013.

[11] A. M. Turing, "The chemical basis of morphogenesis," Philosophical Transactions of the Royal Society of London B, vol. 237, no. 641, pp. 37-72, 1952.

[12] N. F. Britton, Essential Mathematical Biology, Springer, 2003.

[13] G. Sun, Z. Jin, Q.-X. Liu, and L. Li, "Pattern formation in a spatial S-I model with non-linear incidence rates," Journal of Statistical Mechanics, vol. 2007, no. 11, Article ID P11011, 2007.

[14] M. Bendahmane and M. Langlais, "A reaction-diffusion system with cross-diffusion modeling the spread of an epidemic disease," Journal of Evolution Equations, vol. 10, no. 4, pp. 883-904, 2010.

[15] Y. Cai and W. Wang, "Spatiotemporal dynamics of a reactiondiffusion epidemic model with nonlinear incidence rate," Journal of Statistical Mechanics, vol. 2011, no. 2, Article ID P02025, 2011.
[16] W. Wang, Y. Lin, H. Wang, H. Liu, and Y. Tan, "Pattern selection in an epidemic model with self and cross diffusion," Journal of Biological Systems, vol. 19, no. 1, pp. 19-31, 2011.

[17] W.-M. Wang, H.-Y. Liu, Y.-L. Cai, and Z.-Q. Li, “Turing pattern selection in a reaction-diffusion epidemic model," Chinese Physics B, vol. 20, no. 7, Article ID 074702, 2011.

[18] S. Berres and R. Ruiz-Baier, "A fully adaptive numerical approximation for a two-dimensional epidemic model with nonlinear cross-diffusion," Nonlinear Analysis, vol. 12, no. 5, pp. 28882903, 2011.

[19] F. Rao, W. Wang, and Z. Li, "Stability analysis of an epidemic model with diffusion and stochastic perturbation," Communications in Nonlinear Science and Numerical Simulation, vol. 17, no. 6, pp. 2551-2563, 2012.

[20] W. Wang, Y. Cai, M. Wu, K. Wang, and Z. Li, "Complex dynamics of a reaction-diffusion epidemic model," Nonlinear Analysis, vol. 13, no. 5, pp. 2240-2258, 2012.

[21] M. Wang, "Non-constant positive steady states of the Sel'kov model," Journal of Differential Equations, vol. 190, no. 2, pp. 600620, 2003.

[22] P. Y. H. Pang and M. Wang, "Qualitative analysis of a ratiodependent predator-prey system with diffusion," Royal Society of Edinburgh A, vol. 133, no. 4, pp. 919-942, 2003.

[23] P. Y. H. Pang and M. Wang, "Strategy and stationary pattern in a three-species predator-prey model," Journal of Differential Equations, vol. 200, no. 2, pp. 245-273, 2004.

[24] M. Wang, "Stationary patterns for a prey-predator model with prey-dependent and ratio-dependent functional responses and diffusion," Physica D, vol. 196, no. 1-2, pp. 172-192, 2004.

[25] M. Wang, "Stationary patterns caused by cross-diffusion for a three-species prey-predator model," Computers and Mathematics with Applications, vol. 52, no. 5, pp. 707-720, 2006.

[26] R. Peng, J. Shi, and M. Wang, "Stationary pattern of a ratiodependent food chain model with diffusion," SIAM Journal on Applied Mathematics, vol. 67, no. 5, pp. 1479-1503, 2007.

[27] R. Peng, M. Wang, and G. Yang, "Stationary patterns of the holling-tanner prey-predator model with diffusion and crossdiffusion," Applied Mathematics and Computation, vol. 196, no. 2, pp. 570-577, 2008.

[28] R. Peng and J. Shi, "Non-existence of non-constant positive steady states of two Holling type-II predator-prey systems: strong interaction case," Journal of Differential Equations, vol. 247, no. 3, pp. 866-886, 2009.

[29] C. Bianca, "Existence of stationary solutions in kinetic models with gaussian thermostats," Mathematical Methods in the Applied Sciences, vol. 36, no. 13, pp. 1768-1775, 2013.

[30] F. Berezovsky, G. Karev, B. Song, and C. Castillo-Chavez, "A simple epidemic model with surprising dynamics," Mathematical Biosciences and Engineering, vol. 2, no. 1, pp. 133-152, 2005.

[31] C.-S. Lin, W.-M. Ni, and I. Takagi, "Large amplitude stationary solutions to a chemotaxis system," Journal of Differential Equations, vol. 72, no. 1, pp. 1-27, 1988.

[32] Y. Lou and W.-M. Ni, "Diffusion, self-diffusion and crossdiffusion," Journal of Differential Equations, vol. 131, no. 1, pp. 79-131, 1996.

[33] D. Gilbarg and N. S. Trudinger, Elliptic Partial Differential Equations of Second Order, Springer, 1983.

[34] L. Nirenberg, Topics in Nonlinear Functional Analysis, AMS Bookstore, 2001. 


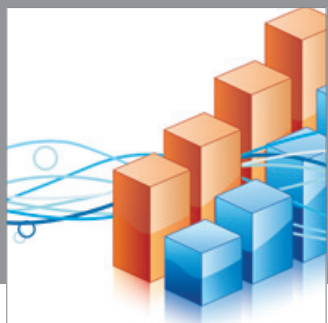

Advances in

Operations Research

mansans

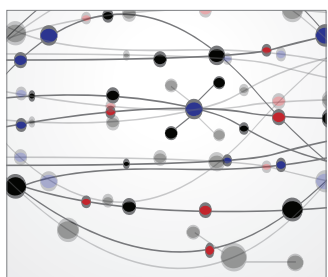

The Scientific World Journal
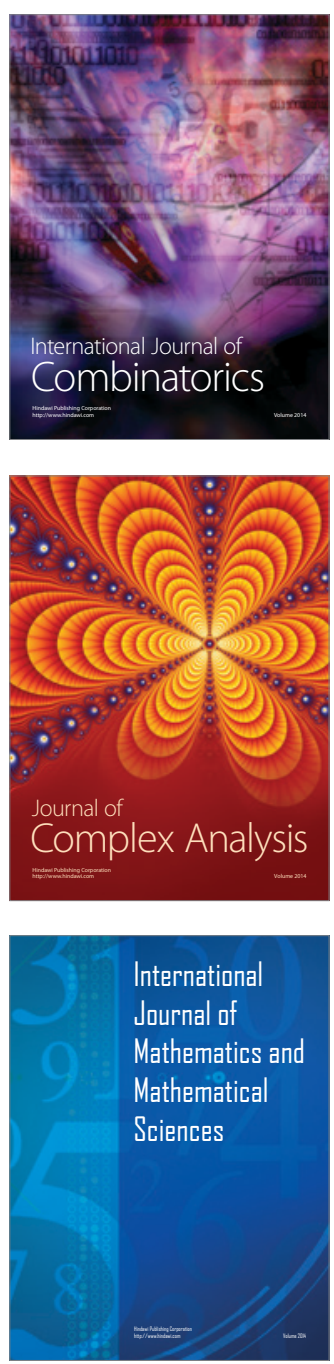
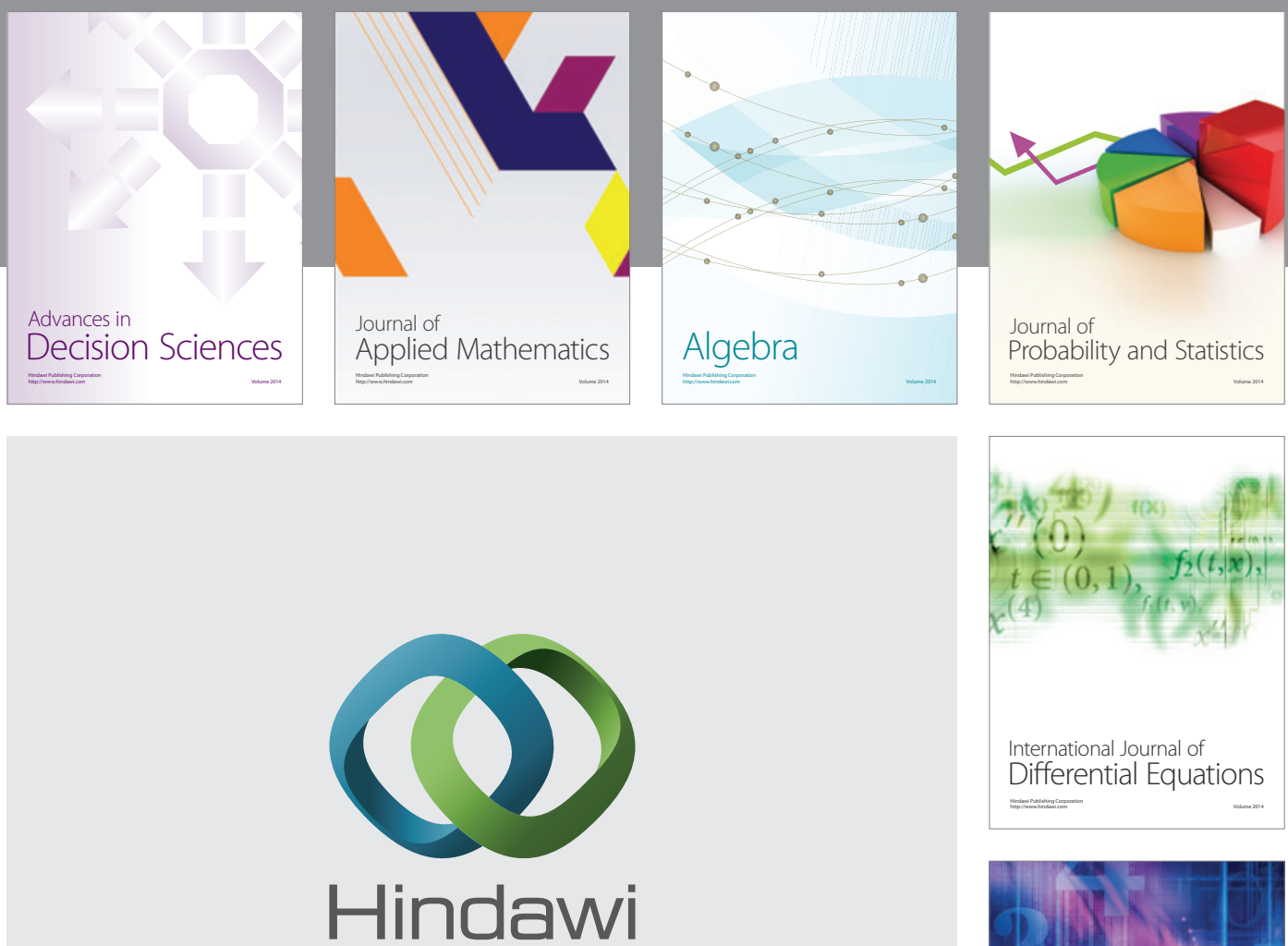

Submit your manuscripts at http://www.hindawi.com
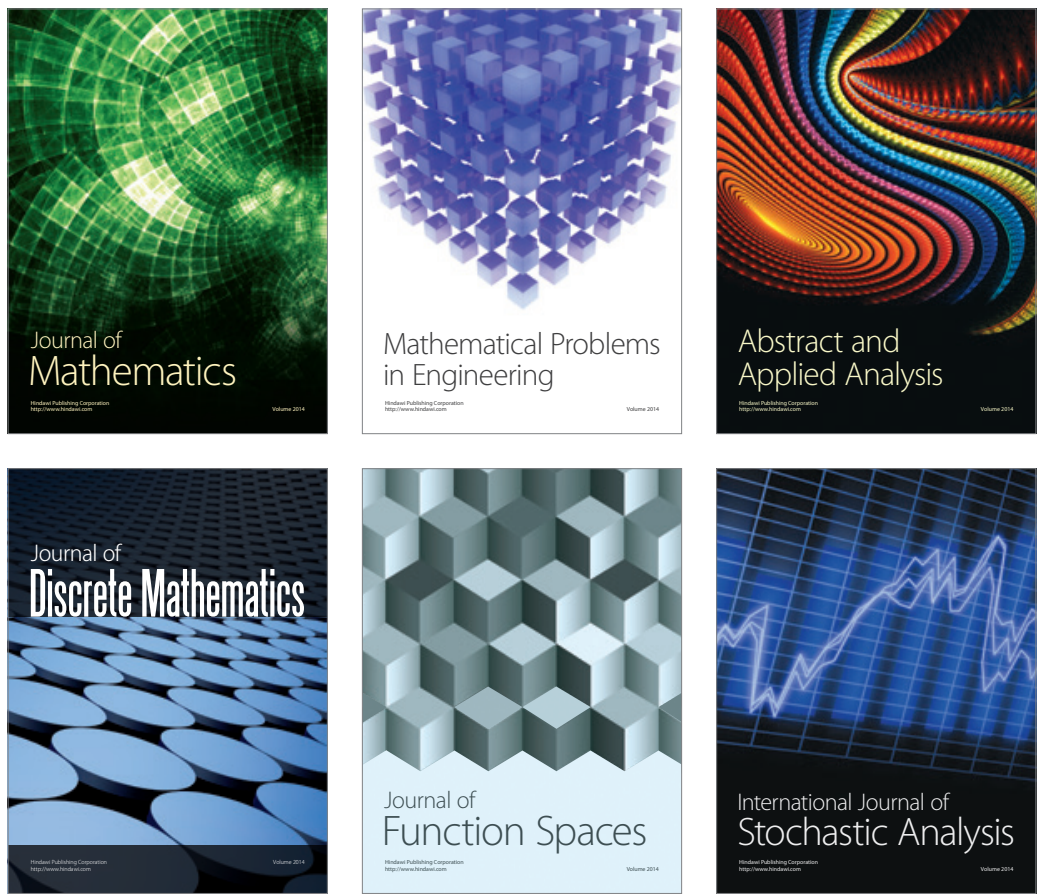

Journal of

Function Spaces

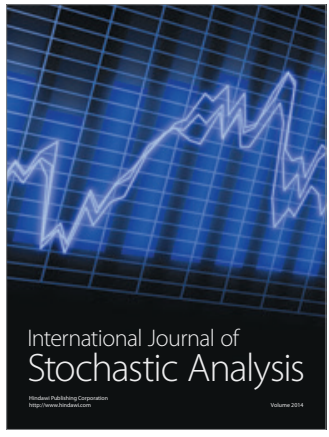

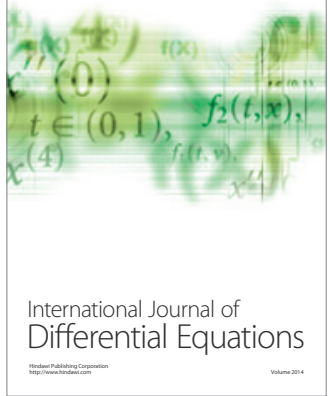
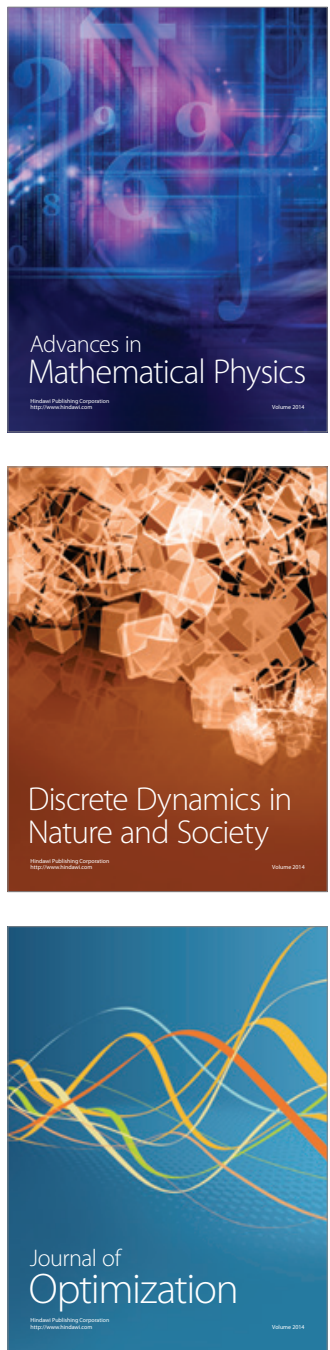